cases though there was suppuration in only one. As there had not been any case of post-operative parotitis in my wards for at least five years before and there has been none since it almost seems as if there must have been some connexion between them; but the patients were not on the same floor or in the same ward and the one died 10 days before the other was operated upon, so that in all probability it was merely a coincidence, but it was a curious one.

CASE 3 - In this case, that of a patient who was under the care of Dr. H. J. Capon, the immediate canse was uncontrollable pain and vomiting. The patient was a married woman, 32 years of age. For some months she had suffered in a very severe degree from all the symptoms of gastric ulcer with the exception of hæmatemesis and melæna. These had never occurred. All the ordinary remedies, including prolonged rectal feeding, had been exhausted without obtaining the least permanent benefit. The patient had lost more than a third of her weight and was so weak that she could scarcely raise herself from her bed. The pain came on within a few minutes after swallowing food and was especially severe at the ensiform cartilage. The skin over this region was exceedingly sensitive. Vomiting shortly after food was the rule. This relieved the worst of the pain but it usually continued with more or less severity for some hours after anything had been taken. After consultation with Dr. 'T. J. Maclagan it was decided to explore the stomach and on opening it a long, narrow ulcer with slightly thickened edges was found lying at the bottom of one of the rugre on the anterior surface close by the incision. It was about threequarters of an inch in length and extended down to, but not into, the muscular coat. No other lesion of any kind could be found though the whole of the interior was carefully explored in the way already described. The ulcer was excised, the mucous and submucous layers were sutured together and the stomach and abdomen were closed in the usual way. In this case, also, vomiting continued for some considerable time. The pain disappeared completely and finally, but owing to the sickness it was some time before the patient could take sufficient food to enable her to regain her strength. Recovery finally was complete.

I may mention that in addition to these cases I published in THE LANGET in $1900^{1}$ a series of three others in which I had operated for hæmatemesis, all of which terminated successfully, the patients recovering completely without any drawback. Of five cases, therefore, in which $I$ have operated directly for hæmatemesis four patients have been cured, and I have little doubt myself that the fifth patient would also have survived had not her condition been so desperate.

It is usually held that the advisability of operating in these cases can be settled at once by comparing the actual mortality of hæmatemesis in cases of gastric ulcer with that which it is supposed might follow gastrotomy performed under these conditions. The former is put down at from 3 to 5 per cent; the latter is estimated very variously, according to the kind and severity of the cases selected. No such comparison, however, is possible under the present conditions. On the one hand, the whole number of cases of hæmatemesis, trivial and severe alike (and the trivial ones are much the more numerous), is taken and the mortality percentage is calculated from that. On the other hand, only those cases which are absolutely desperate, in which the operation is performed as a last chance after everything else has been tried and has failed, are reckoned at all. No one suggests, or has ever suggested, operating upon slight cases of hæmatemesis. They should be placed entirely on one side and not be taken into calculation. The only comparison that can be made with any pretence at fairness is between those cases in which surgical measures are advised and declined and those in which they are advised and accepted. I have no doubt as to the result. In three of my five cases the patients were already so bloodless that transfusion had to be performed. In two others I had to decline to operate altogether; they were beyond even transfusion. The mortality of such cases, left to themselves without operation, is certainly not 3 per cent., or even 5 per cent. ; it is much nearer 100 per cent. And they are the cases with which the only comparison can be made when the result of operation is the subject in question.

As no paper of this kind appears to be complete without statistics, and as the statistics of the mortality from gastric uleer, and especially from hæmatemesis, vary a good deal according to the authority from whom they are taken, I asked the medical registrar at the London Hospital, Dr. R. C. B.
Wall, kindly to tabulate for me all the cases of hæmatemesis from gastric ulcer which had been admitted into the London Hospital in the five years from 1895 to 1899 inclusive. All cases of hæmatemesis from cirrhosis, malignant disease, \&c., were carefully excluded. The result is of decided interest. The total number was 246, 202 women and 44 men, and taking one year with another the numbers were very much the same in each. The total number of deaths from hæmatemesis was 10 , or 4 per cent. But the incidence of the deaths according to age was very singular. Under the age of 30 years there were 153 women with only one death. Over that age there were 49 women with three deaths, or 6 per cent. Taking the men there were only five under 30 years of age with one death, and 39 over 30 years with five deaths. In other words, the mortality from hæmatemesis among the men over 30 years of age is $12 \frac{1}{2}$ per cent., and taking all the men together regardless of age nearly 14 per cent. So far, then, as the question of operation for hæmatemesis is concerned there must, it appears to me, be one rule for women under 30 years of age and another for women over 30 years, and especially for men. But in considering the advisability of operating in general cases of gastric ulcer there is another point that must not be forgotten. These figures are only concerned with the mortality caused by hæmatemesis. There are other causes of death in gastric ulcer besides this, and these causes must also be taken into consideration. When the percentage of deaths due to these is added to that due to hæmatemesis it must be acknowledged that the death-rate due to gastric ulcer, especially in the case of men or of women over 30 years of age, is much higher than is usually believed.

I am fully aware that the treatment of gastric ulcer is, and must remain, in the hands of physicians. But it is becoming more and more evident every day that there are certain contingencies or accidents which may occur in the course of a case of gastric ulcer which, if the consequences are to be avoided, can only be met by the adoption of prompt and decisive surgical measures. This is admitted so far as per foration is concerned, but I maintain that the principle should be carried much farther. It should include all those cases in which, in spite of rectal feeding and other remedies, the pain after food is taken continues with severity, as it did in Case 3; in which vomiting after food obstinately persists; in which the patient is steadily losing ground; and in which medical treatment has been tried thoroughly for a sufficient length of time, say for some months, and has failed.

So far as hæmatemesis is concerned it is clear that age and sex make a very material difference. A much greater risk may be run in the case of a woman under 30 years of age than in the case of those over 30 years, and especially in the case of men. My own figures stand at only one death in 153 cases of hæmatemesis due to supposed gastric ulcer occurring in women under 30 years of age. (I may say that I have reason to believe that that particular period of five years was an exceptionally lucky one.) Surgical treatment can rarely be required in them. But if there is one single severe hæmorrhage in a case in which the previous history suggests the presence of a chronic gastric ulcer (when the bleeding is probably due to an opening in an artery of some size and is not merely capillary); if in any case there are two separate attacks of severe hæmatemesis at a short interval; or if there are frequent small hæmorrhages, so that the patient is becoming seriously anæmic, I believe that the risk of operation is very decidedly less than is the risk of leaving the patient alone, and that the best interests of the patient would be consulted by giving him or her the chance of operation before the condition becomes desperate.

Wimpole-street, $W$.

\section{A FEW OBSERVATIONS ON THE BLOOD PRESSURE IN MENTAL DISEASE,}

\section{WITH A NOTE ON THE TREATMENT OF MELANCHOLIA.}

BY H. DE M. ALEXANDER, M.D. EDIN.,

SENIOR ASSISTANT PHYSTCIAN TO THE ROYAL ASYLUM, ABERDEEN.

THE sphygmometer of Hill and Barnard was used in the following observations and in order to ensure accuracy a duplicate of the instrument was utilised. The readings were taken morning and evening at a fixed hour and the usual 
precautions were observed to obviate any inaccurate result which might arise from the presence of morbid physical conditions liable to produce an abnormal blood pressure. The normal blood pressure was regarded as ranging from 110 to 120 millimetres of mercury.

Melanoholia. - This affection was regarded as being divisible into four varieties-simple melancholia, acute passive melancholia, acute demonstrative melancholia, and chronic melancholia. There was no marked elevation of the blood pressure in the simple form of this malady, whereas in the acute passive type the blood pressure was invariably high and remained so until the acute physical and mental symptoms abated; a re-elevation occurred with each relapse of the disease. The average maximum pulsation was 155 millimetres of mercury.

Fit is probable that the high blood pressure and high tension pulse of this affection are due to the retention in the system of certain waste products of tissue metabolism as evidenced by the marked diminution in the excretion of urea and the atonic condition of the alimentary tract. The subjective physical sensations of the melancholic are probably also due to the same cause.

Di. L. C. Bruce ${ }^{1}$ and myself have already drawn attention to the marked beneficial effect of fluid diet in conjunction with the "bed treatment" in shortening the acute stage of this affection and a shortened acute stage generally ensures a more rapid convalescence.

Since recording these results I have treated 10 more cases of this passive acute melancholia with satisfactory results. No patient undergoing this treatment should leave bed or return to ordinary diet until his blood pressure has remained normal for at least a week or 10 days. I venture to assert that it only requires the further experience and observations of others to prove that the suraliment method of treating acute passive melancholia is unphysiological.

Maudsley ${ }^{2}$ has said regarding the use of drugs in insanity that "the purely medical treatment of insanity which is not maltreatment might be comprised within a narrow compass," and of all types of mental disease his observation is most apulicable to the treatment of the above form of melancholia. Plenty of water, three or four pints of milk daily, the exhibition of an alkaline diuretic which does not disturb digestion-such as citrate of potassium-judicious attention to the bowels, and confinement to bed are all that are required in most cases. General tonics are of little value in the acute stage of the disease. Intestinal disinfectants, such as salol, have been adrocated; but in the acute stage it is probable that they tend injuriously to retard the process of assimilation.

Paraldehyde appears to be the best drug we have at present for the insomnia of this type of melancholia and next to it comes trional. A great objection to sulphonal is that it tends to increase the already existing constipation and thus indirectly to sustain the blood pressure. It is a common experience to see a melancholic, to whom sulphonal has been administered as a hypnotic, improve at once on the withdrawal of the drug and the administration of calomel and salines.

Acute demonstrative melancholia (agitated or excited melancholia) differs from the foregoing passive type physically as well as mentally. Here we have no great diminution in the amount of urine excreted, an excretion of urea little below normal, a less elevated blood pressure, and, in the majority of cases, a lencocytosis. Bodily weight is also lost more rapidly. It is thus, from a physical point of view, more akin to mania.

The blood pressure was taken daily in 14 patients suffering from this variety of melancholia. It followed a more irregular course than that observed in passive melancholia, did not reach such a height, and its descent was not in all cases accompanied by a marked amelioration of the mental symptoms.

The loss of weight, which is usually a pronounced symptom in this affection, is best combated by confinement to bed as long as motor restlessness or marked physical debility is present. The fluid diet treatment is also very beneficial, though it does not require to be so rigidly adhered to as in the former variety of melancholia.

Patients suffering from demonstrative melancholia have nsually been "ill" for some considerable time before they come under asylum treatment: thair malajo is well advancer

1 The LAYCFT, August 24th, 1901, p. 516.

2 Pathology of the Mind. and they, as a rule, take much longer to recover than the passive melancholic.

The employment of opium in the treatment of melancholia is condemned by some authorities and advocated by others. In the acute passive type of the affection opium is certainly contra-indicated by the morbid physical conditions present on the other hand, in demonstrative melancholia opium often gives surprising results, especially in cases where the malady has been existent for some lengthened period. It should be given for its effect on the "mind " and not as a hypnotic if insomnia is a distressing symptom it is best combated by trional given at bedtime; but, speaking generally, if the opium is producing a beneficial effect the employment of this hypnotic will be found unnecessary, sleep gradually returning as the delusions become less omnipotent. We have had better results with nepenthe than with any other preparation of opium or morphia. It should be given in gradually increasing doses and the amount gradually diminished as decided mental improvement is observed. While taking opium the patient should be put on an ordinary full diet and a small dose of calomel given every second or third day.

Trional is the best hypnotic for the insomnia of demonstrative melancholia. To paraldehyde there are two main objections : firstily, it generally requires to be given in an increasing dosage; and secondly, it tends to increase the motor agitation of this affection on the following morning, and more especially so in those patients who have previously been addicted to alcohol.

In chronic melanoholia the blood pressure was generally low; an elevation occurred when the mental symptoms became more acute.

Mania.-Dr. Maurice Craig ${ }^{3}$ found that acute mania was associated with a low pressure, the average blood pressure in 33 cases which he examined being from 105 to 110 millimetres of mercury. Although I have been able to note the blood pressure only in 16 cases of acute mania the reading obtained in each of them was invariably above normal, though it never attained the height observed in acute melancholia. Mental improvement was coincident with its descent though not to such a marked extent as in melancholia. The blood pressure was much lower if exhaustion was present.

In the case of recurrent insanity examination of the pulse suffices to demonstrate the rise in arterial blood pressure which ensues coincidently with the return of excitement. In women recovering from an attack of insanity the onset of the menstrual period is often associated with a recurrence of the previous mental affection, and although the mental disturbance may be only temporary it is, as a rule, accompanied by an increase in the tension of the pulse whether the relapse be characterised by excitement or by depression. The recurrence of excitement in cases of relapsing mania is usually heralded by certain premonitnry symptoms such as insomnia, fits of restlessness, and sometimes by a feeling of general malaise. The blood pressure was observed to be raised from 10 to 20 millimetres of mercury during this prodromal period and it rose to a maximum as the acute attack developed. The "bromide sleep" was induced in a strong adult woman suffering from a very acute attack of mania, by giving three and a balf ounces of sodium bromide in four days. There was no appreciable alteration in her blood pressure as the result of the administration of the drug and no benefit ensued, the maniacal condition returning as the "sleep" passed off. When the attacks of mania and melancholia in folie riroulaire were of the acute type the blood pressure followed the course observed in the acute idiopathic varieties of those affections. The readings obtained in stupor varied according to the variety of the affection. In the anergic and secondary forms the blood pressure was generally subnormal and in no case more than 10 millimetres of mercury above normal ; on the other hand, a much higher reading was obtained in the melancholic resistive variety.

General paralysis presents many types of insanity and the results obtained with the sphygmometer varied extremely, it being only in the later demented stages of the disease that the readings approached uniformity, and the blood pressure was in every case low. No alteration in the arterial pressure was observed in five cases of the disease examined before and after a congestive seizure.

Professor R. Stockman and Dr. F. J. Charteris ${ }^{*}$ have shown

3 The Laxcer, June 25th. 1898. p. 1742, and Brit. Med. Jour.. 1900. $+\mathbf{B}$ it. Med. Jour., 1901. 
that the potassium salts when given in "ordinary quantities" do not usually cause any depressing effect on the heart or on the blood pressure in the arteries, and it would also appear that potassium bromide given in large doses has no depressing effect on the blood pressure in epilepsy, as in 34 cases of epileptic insanity examined a blood pressure of 130 millimetres of mercury was the average reading obtained These patients were taking 90 grains of the drug per diem. In a case of epilepsy in a boy in whom the convulsive attacks were generally nocturnal and came on regularly nearly every day between 8 and 8.30 P.M. no alteration in his blood pressure was observed either before or after the seizure; on the other hand, in an adult woman suffering from the status epilepticus the blood pressure fell from 140 to 90 millimetres of mercury during the short intervals between the seizures-probably as the result of exhaustion.

In pure senile mania and melancholia the readings obtained corresponded to those registered in the same affections occurring in an adult or an adolescent; but the blood pressure was, as a rule, much above normal in that variety of senile insanity which is associated with dementia and which is characterised clinically by a constant fidgety motor restless ness; and it is in this form of insanity that drugs of the nitrite class are of most benefit.

In melancholia we have found the sphygmometer, in conjunction with other clinical methods, of great service in determining what stage of the affection was present and thus indicating the line of treatment to be adopted.

The blood pressure in mania appears to be considerably modified by the presence of general physical debility, and thus restlessness in the chronic maniac of poor physique is as a rule associated with a normal or even low blood pressure, though an elevation may occur with any increase in excitement. Without the presence of any organic disease to account for it some cases of senile dementia with constant restlessness as a marked symptom have a high blood pressure and these patients are often much benefited by the administration of such a drug as erythrol tetranitrate. As is well known an attack of mania is usually ushered in by certain premonitory symptoms and one of the commonest of these is mental depression. On the appearance of any of these premonitory symptoms it is often possible to ward off the acute attack by confining the patient to bed amidst quiet surroundings and by keeping him on a low diet-precisely as a case of melancholia may be treated. I have always found the symptoms, mental and physical, antecedent to an attack of mania to be accompanied by a rise in blood pressure. Dr. Bruce ${ }^{5}$ stopped the onset of excitement or depression in patients labouring under folie circulaire by administering to them the thyroid extract and this agent lowers the blood pressure in addition to increasing metabolism. The sedative and hypnotic effect induced by sulphonal in excitement has also been found to be attended by a reduction of blood pressure.

I believe that mania is associated with a higher blood pressure than that attributed to it by Dr. Craig and Dr. W. R. Dawson ${ }^{6}$ and also that the sedative effect of a hot bath on the excitement of an acute maniac is in part due to the reduction of blood pressure induced by this method of treatment. The "bed treatment" in cases of mania also tends to produce the same result by preserving the patient from sensory and emotional irritation.

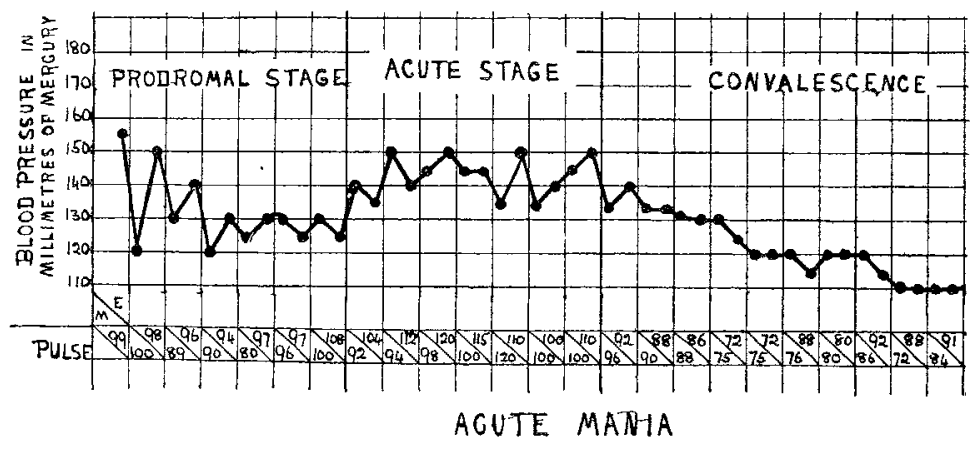

$I$ append a chart illustrating the course of the fblood pressure observed in a case of acute mania which was under observation from the commencement of the affection.

Aberdeen, N.B.

5 Edinburgh Hoapital Reports, 1899, 1900

E Dublin Journal of Mletieal Science, 1900.

\section{VERMIFORM APPENDIX, CONTAINING FECAL CONCRETION ON A BLACK PIN, REMOVED AFTER DEATH FROM A CHILD, AGED SIX YEARS ;}

ILLNESS TWO DAYS AND NO MEDICAL MAN CONSULTED.

\section{By JOHN D. MALCOLM, F.R.C.S. EDIN.,}

SURGEON TO THE SAMARITAN FREE HOSPITAL.

THE concretion which is figured below was exhibited at the meeting of the Pathological Society of London on April 29th, 1902. It was sent to me, with the vermiform appendix in which it was found, by Dr. Percy Rendall of Ewell ; and Dr. C. Hubert Roberts has kindly made the accompanying sketches.

The patient, a female child, whose illness lasted for two days, was not attended by any medical man, but Dr. Rendall was sent for after her death. He was told that the girl was six years old and had shown no evidence of abdominal mischief before Oct. 7th, 1901. On that day she was at school and quite well. In the evening she was seized with

FiG. 1.

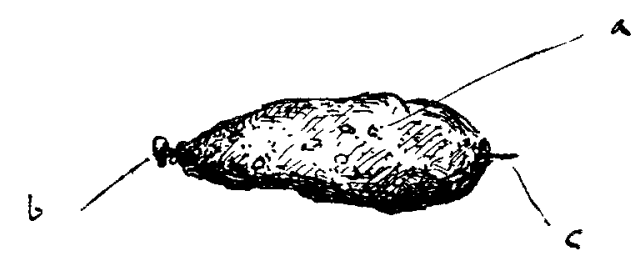

Concretion.- $a$, The concretion. $b$, Head of pin. $c$, Point of pin.

FIG. 2

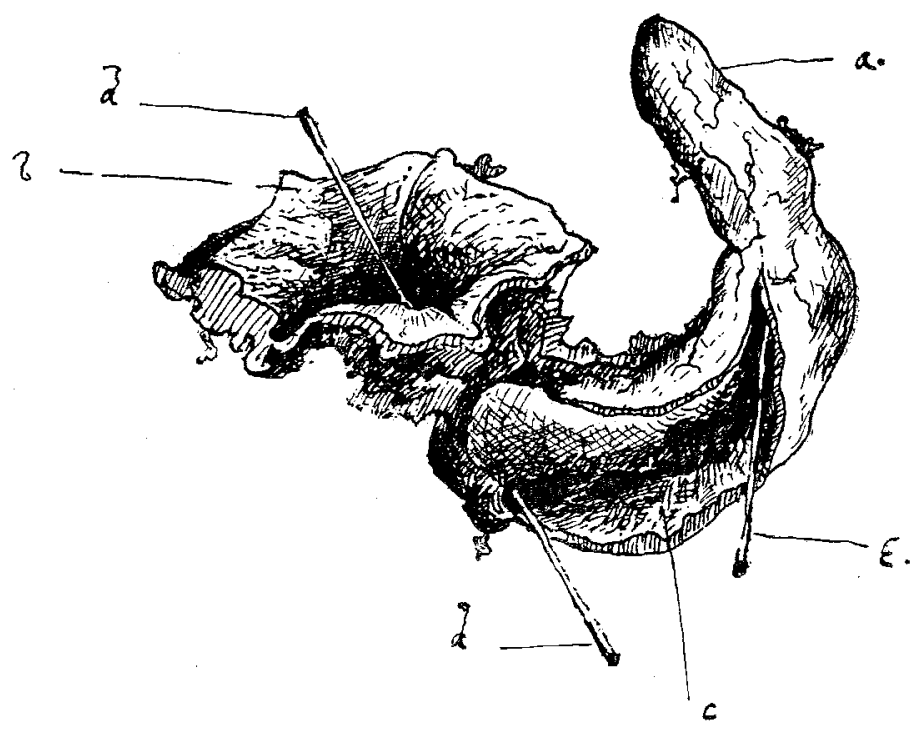

Appendix and part of cæcum.- $-a$. Free end of appendix vermiformis. $b$, Portion of cacum removed with appendix. $c$, Portion of appendix opened up to show region which contained the concretion shown in Fig. 1. d.d. Glass rod passed through from cacum to openinu in appendix. $e$, Glass rod passed into canal towards the blind end of appendix.

violent pain in the abdomen, vomiting, and diarrhoea. The next day she wos better, off and on, but on the 9th she died suddenly. The body was opened by order of the coroner The small intestine and mesentery were found to be glued together by inflammatory adhesions so as to shut off the pelvic cavity. The appendix was in a gangrenous state beneath the adherent coils and close to the peritoneum covering the sacrum. Liquid fæces had exuded and had filled the pelvic cavity. On incising the appendix the con cretion was exposed and it was noted that the point of the pin was directed towards the tip of the appendix. Before the drawing was made the concretion had shrunk so as to expose more of the head and point of the pin than was shown in the fresh moist state.

The case is interesting as it shows that the mrst serious mischief connected with the vermifurm appendix may excite 